\title{
Culture-Dependent Analysis of 16S rRNA Sequences Associated with the Rhizosphere of Lemna minor and Assessment of Bacterial Phenol-Resistance: Plant/Bacteria System for Potential Bioremediation - Part II
}

\author{
Olga Radulović ${ }^{1 *}$, Marija Petriće ${ }^{2}$ Martin Raspor ${ }^{2}$, Olja Stanojević ${ }^{3}$, \\ Tamara Janakiev ${ }^{3}$, Vojin Tadić4, Slaviša Stanković ${ }^{3}$ \\ 'Institute for Chemistry, Technology and Metallurgy, University of Belgrade, Njegoševa 12, Belgrade \\ ${ }^{2}$ Institute for Biological Research "Siniša Stanković", University of Belgrade, \\ Despota Stefana 142 Street, Belgrade, Serbia \\ ${ }^{3}$ Faculty of Biology, University of Belgrade, Studentski Trg 16, Belgrade, Serbia \\ ${ }^{4}$ Mining and Metallurgy Institute, Zeleni Bulevar 35, 19219 Bor, Serbia
}

Received: 27 November 2017

Accepted: 2 January 2018

\begin{abstract}
In this work, we demonstrate that the rhizosphere of common duckweed (Lemna minor) is inhabited with various phenol-resistant bacterial strains. Based on 16S rRNA sequencing, we have identified 60 rhizosphere-associated bacterial isolates belonging to 10 different bacterial genera (Pseudomonas, Hafnia, Serratia, Enterobacter, Micrococcus, Stenotrophomonas, Xanthomonas, Bacillus, Staphylococcus and Klebsiella). All isolates have been tested for phenol resistance and ability to utilize phenol as the sole carbon source. $70 \%$ of all isolates survived high doses of phenol $(\geq 200 \mathrm{mg} / \mathrm{L})$ and at least $27 \%$ can be potentially acclimatized by gradual increase of phenol concentration. Finally, based on high phenol resistance, ability to utilize phenol as the sole carbon source and documented low pathogenicity, we propose 5 strains as potentially excellent candidates for bioremediation. These 5 strains taxonomically correspond to Klebsiella sp., Serratia sp., and Hafnia sp., respectively. To the best of our knowledge, this is the first attempt to assess decontamination capacity of Serratia nematodiphila and Hafnia sp. in the context of bioremediation of phenol-contaminated aqueous media. Although additional analyses are needed, interaction between the common duckweed and the selected bacterial strains may be utilized in future bioremediation strategies.
\end{abstract}

Keywords: bacteria, bioremediation, rhizosphere, phenol resistance

*e-mail: o.radulovic@ihtm.bg.ac.rs 


\section{Introduction}

Phenol contamination is a major environmental and health concern worldwide. Phenol is produced and used in the petrochemical, textile, plastics, and pharmaceutical industries. Therefore it is not surprising that phenol is declared a priority toxic compound in the USA [1]. Although there is no evidence of carcinogenic effects, phenol is a teratogen and an irritant. Phenol concentrations as low as $5 \mathrm{mg} / \mathrm{L}$ are considered toxic to all living organisms, while concentrations of $20 \mathrm{mg} / \mathrm{L}$ and higher are lethal to water vertebrates. Additionally, phenol is most stable and long-lived in aqueous environments, where it can persist for up to 14 days $[1,2]$. A consensus has been reached that the optimal strategy for phenol decontamination of ambient waters includes physical and chemical degradation methods combined with final polishing steps based on the natural ability of some living organisms (primarily bacteria and plants) to neutralize phenol and derived compounds, i.e., bioremediation [2-4]. This is especially true for the aromatic compounds that can still exert adverse effects on the natural environment even years after an incident [5], and aquatic environments are particularly sensitive to this type of pollution due to the fact that phenol is more stable in water than elsewhere, and rapidly dissolves in large volumes of water.

To make the matter even more complex, not all bacterial species are able to degrade phenol or can even survive in a phenol-polluted medium since phenol was not present in significant amounts in the environment until the industrial era. Therefore, bacteria had merely 250 years to adapt, and this evolutionary process is still ongoing [6]. Bacterial species in constant contact with elevated amounts of phenol are under greater selective pressure $[7,8]$, and therefore it is expected that urbanized areas are the source of many phenol-resistant strains, where resistance is defined as the ability of a living organism to survive adverse conditions. In vitro, it is shown that diversity of genes responsible for phenol degradation rises with the phenol increment [8, 9]; furthermore, individual bacterial strains isolated from different microhabitats have been identified as excellent bioremediation agents $[10,11]$; however, little is known about the overall distribution of phenol resistance in specific microhabitats. The rhizosphere can be regarded as a microhabitat adjacent to the plant root where microbial communities are aggregating, attracted by the metabolic activity of the plant, and is also a known place of intense horizontal gene transfer, which helps disseminate resistance-related genes [6-9, 11].

Only highly resistant bacterial strains can be used in bioremediation strategies. Moreover, combining water plants and bacterial communities has been proven to enhance the efficiency of bioremediation $[12$, 13]. Certain species of the Lemnaceae family, such as Spirrodela polyrhiza (a cosmopolitan species) and L. aikoukusa (Japanese species), are known for their ability to eliminate phenol or derived aromatic compounds, with their rhizosphere probably harboring a variety of bacterial species of which only a few with phenol-eliminating activity have been isolated and identified thus far [14-16]. However, investigations regarding the decontamination capacity of Lemna are incomplete because only a small fraction of the family has been analyzed for the potential of phenol-contaminated water. Furthermore, introducing a new species into an ecosystem is always inherently combined with the risk of disturbing the ecological balance or, in the case of bioremediation, of exacerbating the state of an already contaminated site. By selecting a species indigenous to the area where contamination occurred, this risk would be significantly diminished.

The aim of this work was to isolate and identify bacteria from the rhizosphere of a Lemnaceae representative indigenous to Serbia, the common duckweed (Lemna minor, L), grown in a typical urban setting, and to find evidence of bacterial populations with traits that make them good bioremediation candidates.

\section{Experimental Procedures}

\section{Plant Material Preparation and Isolation of Bacteria}

Duckweed was collected from the garden pond of the Siniša Stanković Institute for Biological Research in Belgrade. Pond water is periodically replenished from the communal water supply system. The pond is situated in a typical urban environment, in the centre of the Serbian capital city, and is exposed predominantly to trafficrelated pollution. Duckweed was either brushed gently against sterile filter paper (group A), air dried in an aseptic environment (group B), or washed thoroughly in sterilized distilled water to remove larger contaminants (group D). As a control, $1 \mathrm{~mL}$ of surrounding water was used to inoculate $5 \mathrm{~mL}$ of sterile liquid lysogeny broth (LB) medium (group C) [14]. Cultures were initiated by transferring $100 \mu \mathrm{L}$ of suspension from each LB media to LB solid agar in Petri dishes. The colonies were subcultivated many times to obtain purity. All rounds of cultivation were done at $+27^{\circ} \mathrm{C}$. Colonies were stained according to Gram and checked under the microscope for stain reaction and purity of colonies.

\section{Bacterial DNA Extraction}

Total genomic DNA of selected isolates were prepared according to modified protocol by Le Marrec et al. Briefly, overnight cultures were vortexed, briefly centrifuged at 13,000 $\mathrm{xg}$, and washed in TEN buffer. Lysosime for Gram-positive bacteria or proteinase $\mathrm{K}$ for Gram-negative bacteria were resuspended in PP buffer and added to cell suspension. Cell lysis was performed at $+37^{\circ} \mathrm{C}$ for 30 minutes. The suspension was subsequently centrifuged and the pellet resuspended in TEN buffer. 
Sodium-dodecyl sulphate $(10 \%$ solution) was added and the suspension thoroughly vortexed until transparent. Standard phenol/chloroform extraction ensued. The upper phase was mixed with isopropanol and centrifuged. DNA in pellet was additionally washed and precipitated in $70 \%$ ice-cold ethanol. The samples were left to dry, then resuspended in RNase solution [15].

\section{Polymerase Chain Reaction (PCR) of 16S Ribosomal RNA Genes}

Deleted "good quality" DNA extracts were used in PCR amplification. To amplify 16S ribosomal RNA genes, two universal primers covering the length between positions 8 and 1492 in 16S rRNA genes were used: UN1-16SF (GAGAGTTTGATCCTGGC) and UN1-16SR (AGGAGGTGATCCAGCCG). For amplification we used KAPA Taq DNA Polymerase (KAPA Biosystems, Boston, USA). PCR conditions were adapted according to manufacturer's instructions. PCR products (no overlapping bands, no smearing) were then purified with QIAquick Gel Purification Kit (QIAGEN GmbH, Hilden, Germany), according to manufacturer's instructions. Purified samples were quantified and sent to Macrogen, Netherlands, for sequencing.

\section{Sequence and Statistical Analysis}

Sequences were analyzed by Nucleotide BLAST (blastn) to find the corresponding sequences in the GenBank database. Ferograms were additionally checked visually in FinchTV, version 1.4.0. Best BLAST matches (91\% identity and more) were used as relevant. Phylogenetic trees were constructed in MEGA 7 using the neighbor-joining method. The distances were calculated using the maximum likelihood composite method as the number of base substitutions per site. The topology was evaluated by the bootstrap method with 1000 replicates. Distances were represented as the number of base substitutions per site [16-18]. Bacterial population diversity was quantified through Shannon's diversity index $(\mathrm{H})$ and Simpson's diversity index (D):

$$
\begin{gathered}
H=-\sum_{i}^{N}\left(\frac{n_{i}}{N} \ln \frac{n_{i}}{N}\right) \\
D=\frac{\sum n_{i}\left(n_{i}-1\right)}{N(N-1)}
\end{gathered}
$$

\section{Phenol-Resistance Test}

$100 \mu \mathrm{L}$ liquid LB overnight culture of each isolate was streaked onto a Petri dish containing Murashige and Skoog (MS) agar with sucrose [19]. The MS agar plates were supplemented with 200, 500, and $1000 \mathrm{mg} / \mathrm{L}$ phenol, respectively. Bacteria were grown at $+27^{\circ} \mathrm{C}$ for 5-7 days or until colonies became visible. The colonies were re-streaked from lower to higher concentrations.
Additionally, all isolates were tested immediately from the overnight culture against $1000 \mathrm{mg} / \mathrm{L}$ of phenol in MS agar with sucrose.

\section{Utilizing Phenol as the Sole Carbon Source}

All bacterial strains able to grow on $\geq 200 \mathrm{mg} / \mathrm{L}$ were transferred to aqueous solution of salts from MS recipe with $20 \mathrm{mg} / \mathrm{L}$ of phenol as the sole carbon source. In short, $1 \mathrm{~mL}$ of overnight culture in liquid LB was briefly centrifuged at maximum speed $(13,000 \mathrm{xg})$. Supernatant was discarded and the cells were resuspended in $1 \mathrm{~mL}$ of minimal MS medium. The cultures were grown at $+27^{\circ} \mathrm{C}$ until a significant increase in biomass was observed.

\section{Vitek 2 (Biomerieux) Biochemical Characterization of Selected Isolates}

Five isolates that have previously been shown to multiply abundantly in minimal MS medium with phenol as the sole carbon source and which possess significant resistance to phenol $(\geq 500 \mathrm{mg} / \mathrm{L})$ were biochemically characterized on Vitek 2 (Biomerieux; Mercy, France).

\section{Results and Discussion}

Diversity of Rhizosphere-Associated Bacteria, their Phenol Resistance, and Phenol Utilization

Successful cultivation, isolation, and identification of 60 bacterial strains from the rhizosphere of duckweed (Table 1) were performed. Almost all belong to deleted $\gamma$-Proteobacteria group, except 3 Terrabacteria species (Micrococcus luteus, Staphylococcus sp. and Bacillus sp.). Isolates represent 8 different bacterial families: Pseudomonadaceae deleted (28 isolates), Xanthomonadaceae (7), Hafniaceae (6), Staphylococcaceae (6), Enterobacteriaceae (6), Yersiniaceae (5), Micrococcaceae (1), and Bacillaceae (1). Based on Shannon diversity indices, group A was slightly more diverse, followed by $\mathrm{C}, \mathrm{D}$, and $\mathrm{B}$ (Table 2). Of 60 tested isolates, 18 were unable to grow on $200 \mathrm{mg} / \mathrm{L}$ of phenol, whereas 6 grew on a maximum of $200 \mathrm{mg} / \mathrm{L}$ phenol. Fifteen strains formed colonies at a maximum of $500 \mathrm{mg} / \mathrm{L}$ of phenol and ceased to grow on $1000 \mathrm{mg} / \mathrm{L}$. In total, 21 bacterial strains showed varying degree of growth on a maximum of $1000 \mathrm{mg} / \mathrm{L}$ of phenol. Among these, 5 isolates grew on $1000 \mathrm{mg} / \mathrm{L}$ of phenol after direct inoculation from an overnight culture, i.e., without any prior in vitro acclimatization (Fig. 1; Table 1, non-acclimatized isolates are bolded). Within this group, there were pronounced differences in growth speed and biomass increase. Acclimatized isolates showed toxic effects of phenol and needed approximately 9 days to form. Five strains that were grown without prior acclimatization showed less toxic effects. Of these, Hafnia alvei C31-106/2, 
Table 1. Summary of isolates, their phenol-resistance and ability to grow on phenol as the sole carbon source. (A: duckweeds were brushed against sterile filter paper before transfer to liquid LB medium. B: duckweeds were air-dried in aseptic environment before transfer to LB liquid medium. C: pond water surrounding duckweeds was used to inoculate LB liquid medium. D: duckweeds were washed gently in sterile distilled water and then transferred to LB liquid medium."No" annotates inability to grow on MS solid agar supplemented with 200,500 or $1000 \mathrm{mg} / \mathrm{L}$ of phenol. Further explanations in text.)

\begin{tabular}{|c|c|c|c|c|c|c|c|c|c|c|}
\hline \multirow[b]{2}{*}{ ID } & \multirow[b]{2}{*}{ Strain } & \multirow[b]{2}{*}{ Closest relative } & \multirow{2}{*}{$\begin{array}{l}\text { Access. No. } \\
\text { (closest } \\
\text { relative) }\end{array}$} & \multirow[b]{2}{*}{ Ident. } & \multirow{2}{*}{$\begin{array}{l}\text { Accession No. } \\
\text { (L. minor } \\
\text { rhizosphere } \\
\text { isolates) }\end{array}$} & \multicolumn{4}{|c|}{ Phenol resistance } & \multirow{2}{*}{$\begin{array}{c}\text { Phenol as } \\
\text { the sole } \\
\text { C- source }\end{array}$} \\
\hline & & & & & & No & $\begin{array}{c}200 \\
\mathrm{mg} / \mathrm{L}\end{array}$ & $\begin{array}{c}500 \\
\mathrm{mg} / \mathrm{L}\end{array}$ & $\begin{array}{l}1000 \\
\mathrm{mg} / \mathrm{L}\end{array}$ & \\
\hline 1 & D $4-1-3-100 / 1$ & $\begin{array}{c}\text { Staphylococcus haemolyticus } \\
0078\end{array}$ & KP236214 & $99 \%$ & MF526904 & & + & & & + \\
\hline 2 & D $4-1-3-100 / 2$ & $\begin{array}{c}\text { Staphylococcus haemolyticus } \\
\text { CIFRI P-TSB4 }\end{array}$ & JF784022 & $99 \%$ & MF526905 & & & + & & \\
\hline 3 & D 4-2-100/1 & $\begin{array}{l}\text { Staphylococcus haemolyticus } \\
\text { SS-13 }\end{array}$ & KX964163 & $99 \%$ & MF526906 & & & + & & \\
\hline 4 & D $4-2-100 / 2$ & $\begin{array}{c}\text { Staphylococcus haemolyticus } \\
\text { LCR } 51\end{array}$ & FJ976569 & $99 \%$ & MF526907 & & & + & & \\
\hline 5 & D $4-2-100 / 3$ & $\begin{array}{c}\text { Staphylococcus haemolyticus } \\
\text { strain S1.2.2 }\end{array}$ & KR779793 & $98 \%$ & MF526962 & & & + & & \\
\hline 6 & D 5-1-102 & Enterobacter sp. WJ3 & KT318370 & $100 \%$ & MF536889 & & + & & & \\
\hline 7 & D 6-2-101 & $\begin{array}{c}\text { Bacillus thuringiensis } \\
\text { NBRC3951 }\end{array}$ & AB680181 & $91 \%$ & MF526908 & + & & & & \\
\hline 8 & A5-106/3 & Pseudomonas sp. JL21 & JF740045 & $99 \%$ & MF526909 & & & & + & \\
\hline 9 & A6-104/2 & Klebsiella oxytoca N7 & KM349412 & $99 \%$ & MF526910 & & & & + & + \\
\hline 10 & B1-105/2 & $\begin{array}{c}\text { Enterobacter sp. YUST- } \\
\text { DW17 }\end{array}$ & HM640295 & $94 \%$ & MF526911 & & + & & & + \\
\hline 12 & B2-106/5 & Staphylococcus sp. EA_L_11 & KJ642371 & $98 \%$ & MF526912 & + & & & & \\
\hline 14 & D1-104/2 & Hafnia alvei strain F10 & KT767858 & $99 \%$ & MF526958 & & & & + & \\
\hline 15 & D1-104/3 & $\begin{array}{l}\text { Pseudomonas oryzihabitans } \\
\text { L-1 }\end{array}$ & NR_025881 & $99 \%$ & MF526913 & & + & & & + \\
\hline 16 & D1-105/4 & $\begin{array}{c}\text { Lelliottia amnigena strain } \\
\text { Lmb019 16S }\end{array}$ & KT986089 & $99 \%$ & MF526914 & & + & & & \\
\hline 17 & A3-102/2 & Pseudomonas sp. strain X8 & KY490127 & $99 \%$ & MF526960 & & & + & & + \\
\hline 18 & A3-102/3 & Serratia fonticola GS2 & $\mathrm{CP} 013913$ & $98 \%$ & MF526915 & & & + & & \\
\hline 19 & A3-104/1 & Serratia fonticola GS2 & $\mathrm{CP} 013913$ & $98 \%$ & MF526916 & & & & + & \\
\hline 20 & A3-104/2 & $\begin{array}{c}\text { Stenotrophomonas mal- } \\
\text { tophilia } Q 1\end{array}$ & HE862285 & $99 \%$ & MF526917 & + & & & & \\
\hline 21 & A3-104/3 & Pseudomonas putida KAR35 & KR054997 & $99 \%$ & MF526918 & + & & & & \\
\hline 22 & A3-104/4 & Serratia fonticola GS2 & СР013913 & $98 \%$ & MF526919 & + & & & & \\
\hline 23 & A3-104/5 & Pseudomonas putida L3 & T767824 & $99 \%$ & MF526920 & & & & + & \\
\hline 24 & A5-106/1 & $\begin{array}{l}\text { Pseudomonas oryzihabitans } \\
\text { L-1 }\end{array}$ & NR_025881 & $99 \%$ & MF526921 & & & + & & \\
\hline 25 & A6-104/1 & $\begin{array}{l}\text { Pseudomonas oryzihabitans } \\
\text { L-1 }\end{array}$ & NR_025881 & $98 \%$ & MF526922 & & & & + & \\
\hline 26 & A6-104/3 & $\begin{array}{c}\text { Stenotrophomonas mal- } \\
\text { tophilia Q1 }\end{array}$ & HE862285 & $98 \%$ & MF526923 & + & & & & \\
\hline 27 & $\mathrm{~A} 7-102 / 1$ & Serratia sp. 1136 & JX566540 & $98 \%$ & MF526924 & & & & + & + \\
\hline 28 & A7-102/2 & Pseudomonas sp. E88 & KR703537 & $98 \%$ & MF526925 & + & & & & \\
\hline 29 & B1-104/2 & Pseudomonas putida KAR35 & KR054997 & $99 \%$ & MF526926 & & & + & & + \\
\hline 30 & B1-105/1 & $\begin{array}{c}\text { Pseudomonas brenneri strain } \\
\text { NA }\end{array}$ & KT184488 & $98 \%$ & MF526927 & + & & & & \\
\hline
\end{tabular}


Table 1. Continued.

\begin{tabular}{|c|c|c|c|c|c|c|c|c|c|c|}
\hline 31 & B1-105/3 & Pseudomonas putida KAR35 & KR054997 & $99 \%$ & MF526928 & & & & + & + \\
\hline 32 & B2-106/1 & $\begin{array}{c}\text { Stenotrophomonas mal- } \\
\text { tophilia } Q 1\end{array}$ & HE862285 & $99 \%$ & MF526929 & + & & & & \\
\hline 33 & B2-106/4 & Pseudomonas putida KAR35 & KR054997 & $99 \%$ & MF526930 & & & & + & + \\
\hline 34 & B2-106/6 & $\begin{array}{c}\text { Pseudomonas oryzihabitans } \\
\text { L-1 }\end{array}$ & NR_025881 & $98 \%$ & MF526931 & + & & & & \\
\hline 35 & B3-106/4 & $\begin{array}{c}\text { Stenotrophomonas mal- } \\
\text { tophilia Q1 }\end{array}$ & HE862285 & $99 \%$ & MF526932 & & & + & & + \\
\hline 36 & C31-106/1 & $\begin{array}{c}\text { Hafnia alvei FDAAR- } \\
\text { GOS_158 }\end{array}$ & СР014031 & $99 \%$ & MF526933 & & & & + & \\
\hline 37 & C31-106/2 & Hafnia alvei JCM 1666 & NR_112985 & $99 \%$ & MF526934 & & & & + & + \\
\hline 38 & C31-106/3 & $\begin{array}{c}\text { Pseudomonas gessardii IHBB } \\
9179\end{array}$ & KR085817 & $99 \%$ & MF526935 & + & & & & \\
\hline 39 & C31-106/4 & $\begin{array}{c}\text { Pseudomonas gessardii IHBB } \\
9179 \\
\end{array}$ & KR085817 & $99 \%$ & MF526936 & & & + & & + \\
\hline 40 & C31-106/5 & Pseudomonas fluorescens A12 & KT767652 & $100 \%$ & MF526959 & + & & & & \\
\hline 41 & C32-106/1 & Hafnia alvei 14 & KY849243 & $98 \%$ & MF526937 & & & & + & \\
\hline 42 & C32-106/2 & Pseudomonas sp. AceT-1 & FJ605388 & $99 \%$ & MF526938 & & & & + & + \\
\hline 43 & C32-106/3 & Hafnia paralvei ATCC 29927 & NR_116898 & $99 \%$ & MF526939 & & & & + & + \\
\hline 44 & C32-106/4 & $\begin{array}{c}\text { Pseudomonas fragi NRRL } \\
\text { B-727 }\end{array}$ & LT629783 & $99 \%$ & MF526940 & & & & + & \\
\hline 45 & C32-106/5 & $\begin{array}{c}\text { Pseudomonas putida NBRC } \\
14164\end{array}$ & KY952686 & $98 \%$ & MF526961 & & & + & & \\
\hline 47 & C44-104/1 & $\begin{array}{c}\text { Pseudomonas yamanorum } \\
\text { LMG } 27247\end{array}$ & LT629793 & $99 \%$ & MF526941 & & & & + & + \\
\hline 48 & C44-104/2 & $\begin{array}{l}\text { Hafnia paralvei strain ATCC } \\
29927\end{array}$ & NR_116898 & $98 \%$ & MF526942 & & & + & & \\
\hline 49 & C44-106/1 & Pseudomonas sp. AMF4009 & JQ316310 & $98 \%$ & MF526943 & & & + & & \\
\hline 50 & C44-106/2 & $\begin{array}{c}\text { Enterobacter nimipressuralis } \\
\text { LMG } 10245\end{array}$ & JF430421 & $98 \%$ & MF526944 & & & & + & \\
\hline 51 & D1-104/1 & $\begin{array}{c}\text { Serratia nematodiphila } \\
\text { DZ0503SBS1 }\end{array}$ & NR_044385 & $99 \%$ & MF526945 & & & & + & + \\
\hline 52 & D1-105/1 & Pseudomonas panacis D313 & KT758723 & $98 \%$ & MF526946 & + & & & & \\
\hline 53 & D1-105/2 & Pseudomonas putida KAR35 & KR054997 & $99 \%$ & MF526947 & & & & + & + \\
\hline 54 & $\mathrm{D} 1-105 / 3$ & $\begin{array}{c}\text { Pseudomonas oryzihabitans } \\
\text { IHB B } 13621\end{array}$ & KP762549 & $99 \%$ & MF526948 & & & + & & \\
\hline 55 & D4-106/1 & Pseudomonas putida L3 & $\mathrm{T} 767824$ & $99 \%$ & MF526949 & & & + & & \\
\hline 56 & D4-106/2 & Micrococcus luteus $H N-18$ & KT003262 & $98 \%$ & MF526950 & + & & & & \\
\hline 57 & $5-2-101$ & $\begin{array}{c}\text { Stenotrophomonas mal- } \\
\text { tophilia Q1 }\end{array}$ & HE862285 & $99 \%$ & MF526951 & & + & & & \\
\hline 60 & A3-102/1 & $\begin{array}{c}\text { Xanthomonadales bacterium } \\
\text { F2V8C04 }\end{array}$ & HG322894 & $98 \%$ & MF526952 & + & & & & \\
\hline 62 & B2-106/7 & $\begin{array}{c}\text { Stenotrophomonas sp. LMG } \\
29892 \\
\end{array}$ & KY973973 & $98 \%$ & MF526953 & + & & & & \\
\hline 63 & B3-106/1 & Pseudomonas putida KAR35 & KR054997 & $99 \%$ & MF526954 & & & & + & + \\
\hline 64 & B3-106/2 & Pseudomonas sp. WXBRN2 & KJ184953 & $98 \%$ & MF526955 & + & & & & \\
\hline 65 & B3-106/3 & Klebsiella sp. ZA & KP769536 & $98 \%$ & MF526956 & + & & & & \\
\hline 66 & D12-106 & Pseudomonas putida L3 & T767824 & $99 \%$ & MF526957 & & & & + & + \\
\hline
\end{tabular}


a)

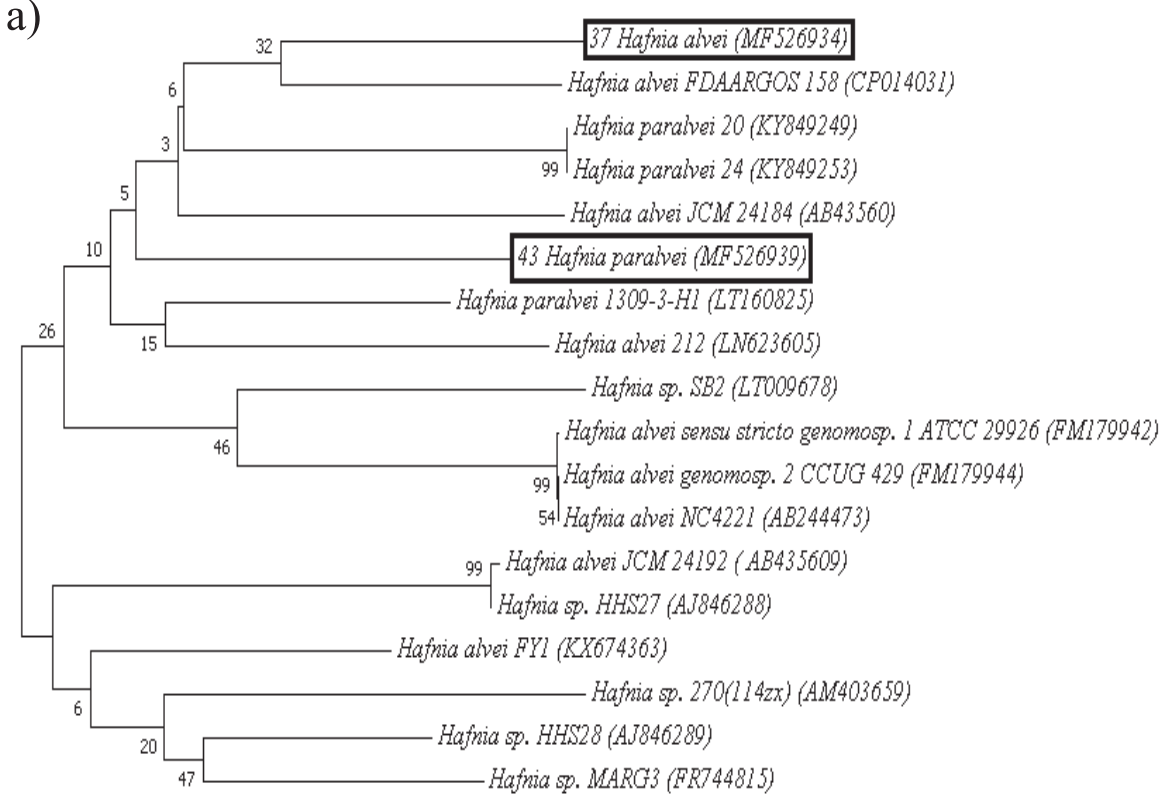

b)

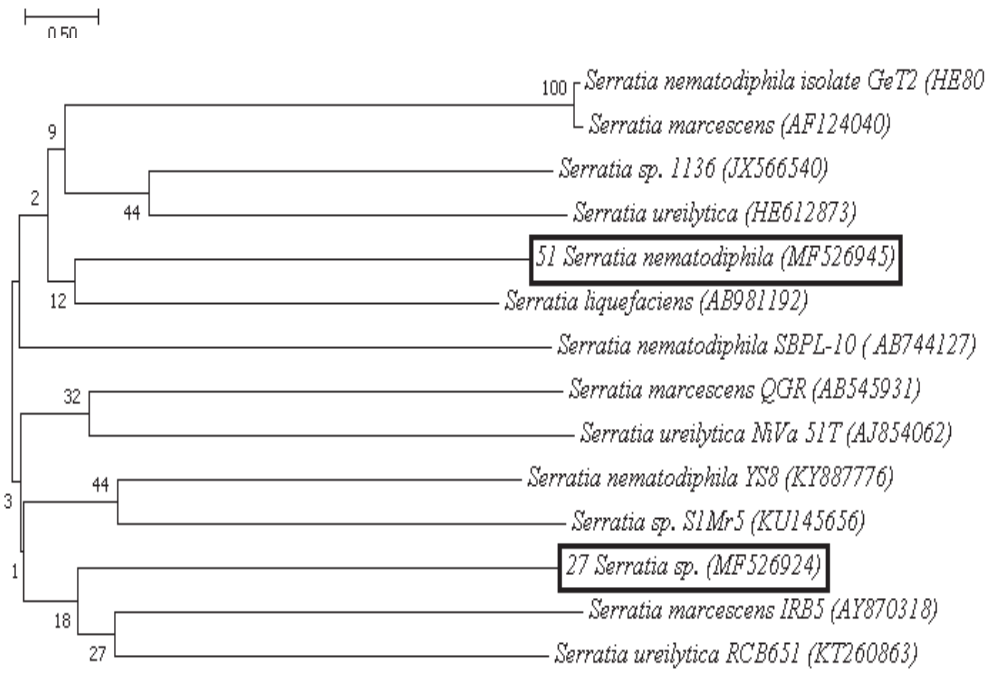

c)

0.20

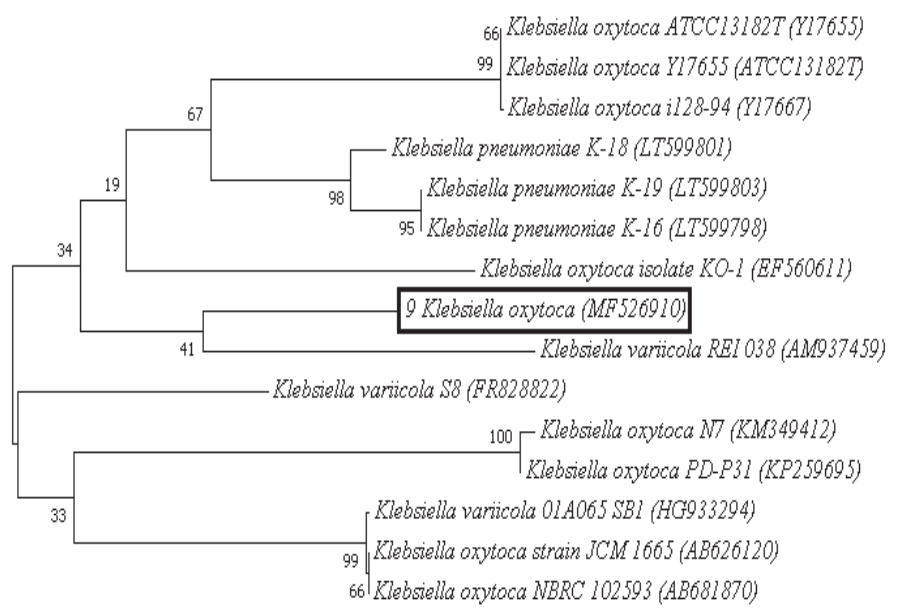

0.50

Fig. 1. Evolutionary relationships of 5 strains proposed as the best phenol-eliminating candidates and related species based on partial $16 \mathrm{~S}$ rRNA sequences (a) - Hafnia, b) - Serratia, c) - Klebsiella; in boxes: 9 Klebsiella oxytoca, 27 Serratia sp., 37 Hafnia alvei, 43 Hafnia paralvei, 51 Serratia nematodiphila). The scale bar represents number of base substitutions per site. 
Table 2. Ecological diversity and phenol-resistance (Shannon's index, H; Simpson's index, D; *defined as ability to grow on MS agar + sucrose supplemented with $200 \mathrm{mg} / \mathrm{L}$ of phenol and more. Additional explanations in text).

\begin{tabular}{|c|c|c|c|c|}
\hline Group & $\begin{array}{c}\text { Shannon's Diversity } \\
\text { Index }\end{array}$ & $\begin{array}{c}\text { Simpson's Diversity } \\
\text { Index }\end{array}$ & $\begin{array}{c}\text { Percentage of phenol } \\
\text { resistant isolates in the } \\
\text { group* }\end{array}$ & $\begin{array}{c}\text { Percentage of isolates able to } \\
\text { use phenol as the sole carbon } \\
\text { source }\end{array}$ \\
\hline A & $2.34 \pm 0.6$ & $0.103 \pm 0.05$ & $60 \% \pm 10 \%$ & $33 \% \pm 1 \%$ \\
\hline B & $2.03 \pm 0.9$ & $0.090 \pm 0.04$ & $54 \% \pm 16 \%$ & $21 \% \pm 11 \%$ \\
\hline C & $2.21 \pm 0.7$ & $0.055 \pm 0.01$ & $86 \% \pm 16 \%$ & $36 \% \pm 4 \%$ \\
\hline D & $2.18 \pm 0.7$ & $0.057 \pm 0.01$ & $82 \% \pm 12 \%$ & $28 \% \pm 4 \%$ \\
\hline Entire population & 2.92 & 0.054 & $70 \%$ & $32 \%$ \\
\hline
\end{tabular}

and Serratia nematodiphila D1-104/1, formed visible colonies after 6 days. Colonies of Klebsiella oxytoca A6-104/2, deleted, became visible after 7 days. Pseudomonas sp. C32-106/2, and Hafnia paralvei C32-106/3, became visible after 8 days of cultivation, but exhibited significantly more detrimental effects of phenol (reduced biomass and retarded growth).

Nineteen strains in total were able to grow on minimal medium with $20 \mathrm{mg} / \mathrm{L}$ phenol as the sole carbon source. However, the most striking biomass increase after 36 hours of cultivation was observed in 5 isolates: $K$. oxytoca A6-104/2, S. nematodiphila D1-104/1, Serratia sp. A7-102/1, H. alvei C31-106/2, and H. paralvei $\mathrm{C} 32-106 / 3$. These strains were characterized by Vitek 2 (Biomerieux, Mercy, France) (Table 3). Serratia sp. A7-102/1 and S. nematodiphila D1-104/1 have been identified as Serratia marcescens (99\%). H. alvei $\mathrm{C} 31-106 / 2$ and $H$. paralvei $\mathrm{C} 32-106 / 3$ have been identified as $H$. alvei (99\%). K. oxytoca A6-104/2 has been identified as $K$. oxytoca $(94 \%)$.

\section{Organization of Bacteria Inhabiting the Rhizosphere of L. minor}

Successful cultivation, isolation, and identification of 60 bacterial strains from the rhizosphere of duckweed (Table 1) were performed. Almost all belong to (deleted) $\gamma$-Proteobacteria group, except 3 Terrabacteria species (Micrococcus luteus, Staphylococcus sp. and Bacillus sp.). Isolates represent 8 different bacterial families: Pseudomonadaceae (deleted: family) (28 isolates), Xanthomonadaceae (7), Hafniaceae (6), Staphylococcaceae (6), Enterobacteriaceae (6), Yersiniaceae (5), Micrococcaceae (1), and Bacillaceae (1). Based on Shannon's diversity indices $(\mathrm{H})$, group A was slightly more diverse, followed by $\mathrm{C}, \mathrm{D}$, and $\mathrm{B}$ (Table 2). Simpson's diversity indices showed more differences in diversity: groups $\mathrm{A}$ and $\mathrm{B}$ were significantly less diverse than groups $\mathrm{C}$ and D. According to Simpson's diversity, the least diverse was group A and the most diverse was group C. Of 60 tested isolates, 18 were unable to grow on $200 \mathrm{mg} / \mathrm{L}$ of phenol, whereas 6 grew on a maximum of $200 \mathrm{mg} / \mathrm{L}$ phenol. Fifteen strains formed colonies at a maximum of $500 \mathrm{mg} / \mathrm{L}$ of phenol and ceased to grow on $1000 \mathrm{mg} / \mathrm{L}$. In total, 21 bacterial strains showed varying degrees of growth on a maximum of $1000 \mathrm{mg} / \mathrm{L}$ of phenol. Among these, 5 isolates grew on $1000 \mathrm{mg} / \mathrm{L}$ of phenol after direct inoculation from an overnight culture, i.e., without any prior in vitro acclimatization (Fig. 1; Table 1, nonacclimatized isolates are bolded). Within this group, there were pronounced differences in growth speed and biomass increase. Acclimatized isolates showed toxic effects of phenol and needed approximately 9 days to form. Five strains that were grown without prior acclimatization showed less toxic effects. Of these, Hafnia alvei C31-106/2, MF526934, and Serratia nematodiphila D1-104/1, formed visible colonies after 6 days. Colonies of Klebsiella oxytoca A6-104/2, became visible after 7 days. Pseudomonas sp. C32-106/2, and Hafnia paralvei C32-106/3, became visible after 8 days of cultivation, but exhibited significantly more detrimental effects of phenol (reduced biomass and retarded growth). Nineteen strains in total were able to grow on minimal medium with $20 \mathrm{mg} / \mathrm{L}$ phenol as the sole carbon source. However, the most striking biomass increase after 36 hours of cultivation was observed in 5 isolates: K. oxytoca A6-104/2, S. nematodiphila D1-104/1, Serratia sp. A7-102/1, H. alvei C31-106/2, and $H$. paralvei C32-106/3. In addition, these strains, proven to thrive in minimal medium with phenol as the sole carbon source and with a significant degree of phenol-resistance $(\geq 500 \mathrm{mg} / \mathrm{L})$, were biochemically characterized by Vitek 2 (Biomerieux, Mercy, France) (Table 3). Sequencing results were in good correlation with results provided by Vitek 2: Serratia sp. A7-102/1 and S. nematodiphila D1-104/1 have been identified as Serratia marcescens (99\%). H. alvei C31-106/2 and $H$. paralvei $\mathrm{C} 32-106 / 3$ have been identified as $H$. alvei (99\%). K. oxytoca A6-104/2 has been identified as K. oxytoca $(94 \%)$.

\section{Organization of Bacteria Inhabiting the Rhizosphere of L. minor}

Slight differences in plant material preparation resulted in visible genetic differences between the isolates (Tables 1 and 2). Groups A and B possess very 
Table 3. Biochemical characterization of bacterial strains that can effectively utilize phenol as the sole carbon source. Characterization was performed on Vitek 2 (Biomerieux; Mercy, France).

\begin{tabular}{|c|c|c|c|c|c|}
\hline Reaction & $\begin{array}{c}\text { Klebsiella oxytoca } \\
\text { (9) }\end{array}$ & $\begin{array}{c}\text { Serratia nematodiphila } \\
\text { (51) }\end{array}$ & $\begin{array}{l}\text { Serratia sp. } \\
\quad(27) \\
\end{array}$ & $\begin{array}{c}\text { Hafnia alvei } \\
\text { (37) }\end{array}$ & $\begin{array}{l}\text { Hafnia paralvei } \\
\text { (43) }\end{array}$ \\
\hline Ala-Phe-Pro-Arylamidase & - & - & - & - & - \\
\hline $\mathrm{H} 2 \mathrm{~S}$ production & - & - & - & + & - \\
\hline Beta-glucosidase & + & + & + & - & - \\
\hline L-Pro-Arylamidase & + & + & + & - & + \\
\hline Saccharose & + & + & + & - & - \\
\hline L-Lactate Alcalinisation & + & + & + & + & + \\
\hline Glycine Arylamidase & $(+)$ & + & + & - & - \\
\hline O129 Resistance (vibrio comp.) & + & + & + & - & + \\
\hline Adonitol & + & + & + & + & - \\
\hline Beta-N-Acetyl-Glucosaminidase & - & + & + & + & + \\
\hline D-Maltose & + & - & + & - & + \\
\hline Lipase & - & - & - & - & - \\
\hline D-tagatose & + & - & - & - & - \\
\hline Alpha Glucosidase & - & - & - & + & - \\
\hline Ornithine Decarboxylase & - & + & + & - & + \\
\hline Glu-Gly-Arg Aminidase & - & + & + & - & - \\
\hline L-Pyrrolidonil Arylamidase & + & + & + & - & - \\
\hline Glutamyl arylamidase pNA & - & - & - & + & - \\
\hline D-Mannitol & + & + & + & - & + \\
\hline Palatinose & + & - & - & + & - \\
\hline D-trehalose & + & + & + & + & + \\
\hline Succinate alcalinisation & + & + & - & + & + \\
\hline Lysine decarboxylase & + & + & + & - & + \\
\hline L-malate assimilation & $(-)$ & - & - & - & - \\
\hline L-arabitol & - & + & + & + & - \\
\hline D-glucose & + & + & + & + & + \\
\hline D-mannose & + & + & + & + & + \\
\hline Tyrosine arylamidase & + & + & + & - & + \\
\hline Citrate & + & + & + & - & - \\
\hline Beta-N-Acetyl-Galactosaminidase & - & - & + & - & - \\
\hline L-Histine Assimilation & - & - & - & + & - \\
\hline ELLMAN & - & - & - & - & + \\
\hline D-cellobiose & + & - & - & + & - \\
\hline Gamma-Glutamyl-Transferase & + & + & - & - & + \\
\hline Beta-Xylosidase & + & - & - & - & - \\
\hline Urease & + & - & - & + & - \\
\hline Malonnate & + & - & - & - & + \\
\hline Alpha-Galactosidase & + & - & - & - & - \\
\hline
\end{tabular}


Table 3. Continued.

\begin{tabular}{|c|c|c|c|c|c|}
\hline Coumarate & - & + & + & - & - \\
\hline L-Lactate assimilation & + & - & - & - & - \\
\hline Beta-Galactosidase & + & + & + & - & + \\
\hline Fermentation of glucose & + & + & + & - & + \\
\hline Beta-Alanine Arylamidase & - & + & + & - & - \\
\hline D-Sorbitol & + & - & - & + & - \\
\hline 5-ketogluconate & + & + & + & - & + \\
\hline Phosphatase & - & - & - & + & + \\
\hline
\end{tabular}

similar Simpson's diversity indices, with the number of phenol-resistant isolates appearing to be reduced in comparison with $\mathrm{C}$ and D (Table 2). It appears that drying out and brushing off of plant material in groups A and B significantly diminishes the diversity of the organisms found in the association with the root, as observed with Simpson's diversity indices and to some extent with Shannon's indices. Interestingly, group C contains predominantly Pseudomonas sp. and Hafnia sp. strains that are restricted to this group only (Table 1, section C). The complete disjunction in taxonomic composition between group $\mathrm{C}$ and groups $\mathrm{A}$, $\mathrm{B}$, and D suggests that group C, obtained by cultivation of bacteria from the pond water, contains bacterial species and strains that are not associated at all with the roots of L. minor, while groups A, B, and D contain bacterial strains that might be associated with the rhizosphere of Lemna to a varying extent. Moreover, distribution of species and strains across the groups suggests that rhizosphere population is not disorganized, but rather specific, and most probably based on chemotaxis, since almost all bacteria associated with the rhizosphere are known to be actively motile. Based on both Shannon and Simpson's indices, the diversity of each group is diminished compared to the overall ecological diversity of the whole rhizosphere-associated population. This also reinforces further the hypothesis that various bacteria prefer the proximity of the plant root. The rhizosphere attracts bacteria primarily by the phenolic exhudates of the plant [24-26], which is an effective explanation of why phenol resistance is so frequent within the rhizosphereassociated bacteria.

Finding an Appropriate Bacterial Candidate or Bioremediation of Phenol-Contaminated Waters: Correlation with the Current Scientific Literature

Phenol exerted the expected toxic effect on bacteria, although a remarkably high number of isolates (70\%) survived beyond the $200 \mathrm{mg} / \mathrm{L}$ point, which is considered a highly toxic and even lethal concentration to the living organisms $[1,2]$. Widely distributed phenol resistance is probably attributable to the fact that the sampling site (the pond) is located in an urban area close to an industrial zone notorious for persistent and high above average values of particulate matter of less than 10 microns in diameter $\left(\mathrm{PM}_{10}\right)$ and soot pollution as the consequence of intensive traffic and coal-tar use during winter [27]. Five isolates were able to grow on $1000 \mathrm{mg} / \mathrm{L}$ of phenol without any prior acclimatization, while 16 continued to grow on $1000 \mathrm{mg} / \mathrm{L}$ of phenol after streaking and restreaking from lower concentrations, which led to at least some degree of acclimatization. Among tested isolates, we have selected $H$. alvei, $H$. paralvei, Serratia sp., $S$. nematodiphila, and $K$. oxytoca as the best candidates for further analyses based on the exhibited biomass increase in minimal medium and high phenol resistance. $K$. oxytoca probably survived due to its documented phenol biodegradation activity [28, 29]. Additionally, the species is living as an endophyte and a plant growth-promoting bacterium, as has been documented in the case of related strain K. oxytoca RS-5 [29]. Plant growth promoting ability is seemingly not uncommon in $K$. oxytoca species [30]. Serratia nematodiphila is also a documented endophyte that synthesizes indol-acetic acid (IAA), among other plant growth-promoting hormones [31]. Moreover, closely related species can degrade substituted monocyclic phenols [32]. However, to the best of our knowledge, data concerning phenol decontamination capacity of $S$. nematodiphila are lacking. Regarding Hafnia sp., two closely related $H$. alvei and the novel $H$. paralvei, apart from living in symbiosis with the plant, also commonly create biofilm, which can explain the phenol resistance, although the literature data describing phenol elimination is apparently lacking as in the case of Serratia sp. [32-35]. Almost all of these bacterial species are opportunistically pathogenic (rare causative agents of infections and/or related to infections of heavily immunocompromised individuals only), and commensal (widespread in the natural environment), which makes them comparatively safe for use in potential bioremediation [36]. The exception is the relatively newfound species of $S$. nematodiphila, which has not yet been associated with any case of infection in humans and invades only insects and their larvae mainly due to a specific mutation of their serralysin protein [37]. Finally, the predominant genus 
Pseudomonas has a well-documented biodegradation capacity, and its representatives are generally known as "the cleaners" of the natural environment. One of our isolates, Pseudomonas sp. C32-106/2, was able to grow directly on MS agar supplemented with $1000 \mathrm{mg} / \mathrm{L}$ phenol, albeit weaker in comparison with the remaining isolates. The most prevalent among our isolates was Pseudomonas putida strain KAR35, associated with various rhizobacterial communities [38]. Although $P$. putida has been extensively used as a model organism for studies of phenol degradation kinetics, surprisingly, in our study Hafnia sp., Serratia sp., and Klebsiella sp. showed considerably greater phenol-resistance and an ability to grow on phenol as the sole carbon source $[39,40]$. This possibly reflects the specific roles certain strains might acquire over time while occupying a very specific niche within an ecosystem. The vast majority of the efficient phenol degraders has been conventionally isolated from the sites of catastrophic phenol pollution (activated sludge, heavily contaminated coal mine soil, municipal and industrial wastewaters) - however, in this work we demonstrate that considerable phenol-degrading activity and resistance can be found in communal waters of urban surroundings and that the bacterial phenol degraders are diverse and organized within the rhizosphere [41]. The bacteria we identified might be best suited for the lesser but recalcitrant pollution since their resistance to phenol did not exceed $1000 \mathrm{mg} / \mathrm{L}$. On the other hand, the bacterial association with a photosynthesizing, non-invasive macroscopic species might contribute to the quicker biological recovery of the contaminated water. Indeed, the data concerning phenoldegradation and phenol-tolerance of European ecotypes of $L$. minor is largely absent from the scientific literature. Therefore, this research is an early attempt to introduce European (Serbian) ecotype of L. minor and its specific rhizosphere bacterial population into the bioremediation of phenolic contamination.

\section{Conclusions}

Rhizosphere of the common duckweed (L. minor L.) grown in urban ponds is a natural source of different phenol-resistant bacteria, which reflects the adaptation to urbanization of their natural habitat. Phenol resistance is a condicio sine qua non for potential bioremediation candidates. Furthermore, we observed that bacterial rhizosphere-associated communities are not randomly distributed, but visibly organized. This fact is reflected in different population structures relative to plant material preparation and in the striking differences between the free-water populations and rhizosphere populations. It is also worth noting that acclimatization is indeed possible for a significant number of isolated strains. Based on their well-documented low pathogenicity, ability to grow on phenol as the sole carbon source, phenol-resistance and biomass increase in minimal liquid medium, we have selected 5 strains as potentially excellent candidates for bioremediation. To the best of our knowledge, this is the first mention of $S$. nematodiphila, H. alvei, and $H$. paralvei in the context of biological decontamination of phenol-polluted waters. Additional investigations of the dynamics of phenol elimination with a dual plantbacterial system are needed. However, the utilization of aforementioned bacterial cultures attached with the root of duckweed in the putative bioremediation strategies seems promising.

\section{Acknowledgements}

The authors would like to thank Zlatica Čakić, MD, for her unselfish support and constructive criticism. This research was financed by the Serbian Ministry of Science and Education, project No. TR37001.

\section{Conflict of Interest}

The authors declare no conflict of interest.

\section{References}

1. Agency for Toxic Substances and Disease Registry (ATSDR). Toxicological profile for Phenol. Atlanta, GA: U.S. Department of Health and Human Services, Public Health Service. Downloaded from: www.atsdr.cdc.gov/ toxprofiles/tp.asp?id=148\&tid=27. 2008.

2. PRADEEP N.V., ANUPAMA S., NAVYA K., ET AL. Biological removal of phenol from wastewaters: a mini review. Appl. Water Sci. 5: 105, 2015.

3. HARBOTTLE M., LEAR G. Bioremediation: Current Research and Emerging Technologies. Norfolk, UK: Caister: Academic Press, 2016.

4. OLLER I., MALATO S., SÁNCHEZ-PÉREZ J.A. Combination of Advanced Oxidation Processes and biological treatments for wastewater decontamination - a review. Sci. Total. Environ. 409 (20), 4141, 2011.

5. RUSIN M., GOSPODAREK J., NADGÓRSKA-SOCHA A. The Effect of Petroleum-Derived Substances on the Growth and Chemical Composition of Vicia faba L. Pol. J. Environ. Stud. 24 (5), 2157, 2015.

6. DE LA CRUZ F., DAVIES J. Horizontal gene transfer and the origin of species: lessons from bacteria. Trends Microbiol. Review. 8 (3), 128, 2000.

7. TAVITA K., MIKKEL K., TARK-DAME M., JERABEK H., TERAS R., SIDORENKO J., TEGOVA R., TOVER A., DAME R.T., KIVISAAR M. Homologous recombination is facilitated in starving populations of Pseudomonas putida by phenol stress and affected by chromosomal location of the recombination target. Mutat. Res. 737 (1-2), $12,2012$.

8. COPLEY S.D. Evolution of a metabolic pathway for degradation of a toxic xenobiotic: the patchwork approach. Trends Biochem. Sci. Review. 25 (6), 261, 2000.

9. SILVA C.C., HAYDEN H., SAWBRIDGE T., MELE P., DE PAULA S.O., SILVA L.C., VIDIGAL P.M., VICENTINI R., SOUSA M.P., TORRES A.P., SANTIAGO V.M., OLIVEIRA V.M. Identification of genes and pathways related to phenol degradation in metagenomic libraries 
from petroleum refinery wastewater. PLoS One. 8 (4), e61811, 2013.

10. BASILE L.A., ERIJMAN L. Maintenance of phenol hydroxylase genotypes at high diversity in bioreactors exposed to step increases in phenol loading. FEMS Microbiol. Ecol. 73 (2), 336, 2010.

11. KOPMANN C., JECHALKE S., ROSENDAHL I., GROENEWEG J., KRÖGERRECKLENFORT E., ZIMMERLING U., WEICHELT V., SIEMENS J., AMELUNG W., HEUER, H., SMALLA K. Abundance and transferability of antibiotic resistance as related to the fate of sulfadiazine in maize rhizosphere and bulk soil. FEMS Microbiol. Ecol. 83 (1), 125, 2013.

12. CHEN W.M., TANG Y.Q., MORI K., WU X.L. Distribution of culturable endophytic bacteria in aquatic plants and their potential for bioremediation in polluted waters. Aqua Biol. 15 (2), 99, 2012.

13. GAŁĄZKA A., KRÓL M., PERZYŃSKI A. The Efficiency of Rhizosphere Bioremediation with Azospirillum sp. and Pseudomonas stutzeri in Soils Freshly Contaminated with PAHs and Diesel Fuel. Pol. J. Environ. Stud. 21 (2), 345, 2012.

14. KRISTANTI R.A., KANBE M., TOYAMA T., TANAKA Y., TANG Y., WU X., MORI K. Accelerated biodegradation of nitrophenols in the rhizosphere of Spirodela polyrrhiza. J. Environ. Sci. 24 (5), 800, 2012.

15. TOYAMA T., SEI K., YU N., KUMADA H., INOUE D., HOANG H, SODA S., CHANG Y.C., KIKUCHI S., FUJITA M., IKE M. Enrichment of bacteria possessing catechol dioxygenase genes in the rhizosphere of Spirodela polyrrhiza: a mechanism of accelerated biodegradation of phenol. Water Res. 43 (15), 3765, 2009.

16. YAMAGA F., WASHIO K., MORIKAWA M. Sustainable biodegradation of phenol by Acinetobacter calcoaceticus P23 isolated from the rhizosphere of duckweed Lemna aoukikusa. Environ. Sci. Technol. 44 (16), 6470, 2010.

17. BERTANI G. Lysogeny at Mid-Twentieth Century: P1, P2, and Other Experimental Systems. J. Bacter. 186 (3), 595, 2004.

18. LE MARREC C., HYRONIMUS B., BRESSOLLIER P., VERNEUIL B., URDACI M.C. Biochemical and genetic characterization of coagulin, a new antilisterial bacteriocin in the pediocin family of bacteriocins, produced by Bacillus coagulans I(4). Appl. Environ. Microbiol. 66 (12), $5213,2000$.

19. SAITOU N., NEI M. The neighbor-joining method: A new method for reconstructing phylogenetic trees. Mol. Bio. Evo. 4, 406, 1987.

20. TAMURA K., NEI M., AND KUMAR S. Prospects for inferring very large phylogenies by using the neighborjoining method. Proceedings of the National Academy of Sciences (USA). 101 (19), 11030, 2004.

21. KUMAR S., STECHER G., TAMURA K. MEGA7: Molecular Evolutionary Genetics Analysis version 7.0 for bigger datasets. Mol. Bio. Evo. 33, 1870, 2016.

22. PIETRINI F., DI BACCIO D., ACEÑA J., PÉREZ S., BARCELÓ D., ZACCHINI M. Ibuprofen exposure in Lemna gibba L.: Evaluation of growth and phytotoxic indicators, detection of ibuprofen and identification of its metabolites in plant and in the medium. J. Hazard. Mat. 300, 189, 2015.

23. HADIA F., AMBREEN A. How Chromium-Resistant Bacteria Can Improve Corn Growth in ChromiumContaminated Growing Medium. Pol. J. Environ. Stud. 25 (6), 2357, 2016.
24. LONG H.H., SONNTAG D.G., SCHMIDT D.D., BALDWIN, I. T. The structure of the culturable root bacterial endophyte community of Nicotiana attenuata is organized by soil composition and host plant ethylene production and perception. New Phytologist. 185 (1), 554, 2010.

25. NEAL A.L., AHMAD S., GORDON-WEEKS R., TON J. Benzoxazinoids in root exudates of maize attract Pseudomonas putida to the rhizosphere. PLoS One. 7 (4), e35498, 2012.

26. DOORNBOS R.F., VAN LOON L.C., BAKKER P.A.H.M. Impact of root exudates and plant defense signaling on bacterial communities in the rhizosphere. A review. Agron. Sustain. Dev. 32 (1), 227, 2012.

27. Plan of air-quality in agglomeration Belgrade - city of Belgrade. Available at: www.beograd.rs/images/data/8e3 32b627c975c8c20bd56adc3dd5c0b_5051701029.pdf. 23, Serbia, 2016.

28. DASTAGER S.G., DEEPA C.K., PANDEY A. Isolation and characterization of high-strength phenol-degrading novel bacterium of the Pantoea genus. Bioremed. J., 13 (4), 171, 2009.

29. WU Z., PENG Y., GUO L., LI C. Root colonization of encapsulated Klebsiella oxytoca RS-5 on cotton plants and its promoting growth performance under salinity stress. Europ. J. Soil Biol. 60, 81, 2014.

30. SINGH R., JHA P., JHA P.J. The plant-growth-promoting bacterium Klebsiella sp. SBP-8 confers induced systemic tolerance in wheat (Triticum aestivum) under salt stress. J. Plant Physiol. 184, 57, 2015.

31. DASTAGER S.G., DEEPA C.K., PANDEY A. Potential plant growth-promoting activity of Serratia nematodiphila NII-0928 on black pepper (Piper nigrum L.). World J. Microbiol. Biotechnol. 27 (2) 259, 2011.

32. SINGH S., SINGH B.B., CHANDRA R. Synergistic biodegradation of pentachlorophenol by Bacillus cereus (DQ002384), Serratia marcescens (AY927692) and Serratia marcescens (DQ002385). World J. Microbiol. Biotechnol. 25 (1), 1821, 2009.

33. HUYS G., CNOCKAERT M., ABBOTT S.L., JANDA J.M., VANDAMME P. Hafnia paralvei sp. nov., formerly known as Hafnia alvei hybridization group 2. Int. J. Syst. Evol. Microbiol. 60 (8), 1725, 2009.

34. VIANA E.S., CAMPOS M.E., PONCE A.R., MANTOVANI H.C., VANETTI M.C. Biofilm formation and acyl homoserine lactone production in Hafnia alvei isolated from raw milk. Biol. Res. 42 (4), 427, 2009.

35. JOHNSTON-MONJE D., RAIZADA M.N. Conservation and Diversity of Seed Associated Endophytes in Zea across Boundaries of Evolution, Ethnography and Ecology. PLoS ONE. 6 (6), 203, 2011.

36. BERG G., EBERL L., HARTMANN A. The rhizosphere as a reservoir for opportunistic human pathogenic bacteria. Environ. Microbiol. Review. 7 (11), 1673, 2005.

37. ZHANG C.X., YANG S.Y., XU M.X., SUN J., LIU H., LIU J.R., ZHANG K.Y. Serratia nematodiphila sp. nov., associated symbiotically with the entomopathogenic nematode Heterorhabditidoides chongmingensis (Rhabditida: Rhabditidae). Internat. J. Syst. and Evo. Microbiol. 59 (7), 1603, 2009.

38. NEAL A.L., AHMAD S., GORDON-WEEKS R., TON J. Benzoxazinoids in root exudates of maize attract Pseudomonas putida to the rhizosphere. PLoS ONE. 7 (4), 1, 2012. 
39. HASAN S.A., JABEEN S. Degradation kinetics and pathway of phenol by Pseudomonas and Bacillus species. Biotech, Biotech. Equip. 29 (1), 45, 2015.

40. KURZBAUM E., KIRZHNER F., SELA S., ZIMMELS Y., ARMON R. Efficiency of phenol biodegradation by planktonic Pseudomonas pseudoalcaligenes (a constructed wetland isolate) vs. root and gravel biofilm. Water Research, 44 (17), 5021, 2010.

41. GU Q., WU Q., ZHANG J., GUO W., WU H., SUN M. Community analysis and recovery of phenol-degrading bacteria from drinking water biofilters. Front. Microbiol. 7, 495, 2016. 